\title{
Erratum: Off-forward gluonic structure of vector mesons [Phys. Rev. D 95, 114515 (2017)]
}

\author{
W. Detmold, D. Pefkou, and P.E. Shanahan
}

(Q) (Received 16 January 2019; published 29 January 2019)

DOI: 10.1103/PhysRevD.99.019904

This erratum corrects Eqs. (7) and (8) and the unnumbered equation in Appendix B. Note that these corrections are to the generalizations of these equations beyond those which are studied numerically and, correspondingly, the numerical results presented in the original manuscript remain unchanged.

Equation (7) should be replaced by [1]

$$
\begin{aligned}
& \left\langle p^{\prime} E^{\prime}\left|S\left[G_{\mu \alpha} i \stackrel{\leftrightarrow}{D}_{\mu_{1}} \ldots i \stackrel{\leftrightarrow}{D}_{\mu_{n}} G_{\nu}{ }^{\alpha}\right]\right| p E\right\rangle=\sum_{\substack{m \text { even } \\
m=0}}^{n}\left\{B_{1, m}^{(n+2)}\left(\Delta^{2}\right) M^{2} S\left[E_{\mu} E_{\nu}^{*} \Delta_{\mu_{1}} \ldots \Delta_{\mu_{m}} P_{\mu_{m+1}} \ldots P_{\mu_{n}}\right]\right. \\
& +B_{2, m}^{(n+2)}\left(\Delta^{2}\right) S\left[\left(E \cdot E^{\prime *}\right) P_{\mu} P_{\nu} \Delta_{\mu_{1}} \ldots \Delta_{\mu_{m}} P_{\mu_{m+1}} \ldots P_{\mu_{n}}\right] \\
& +B_{3, m}^{(n+2)}\left(\Delta^{2}\right) S\left[\left(E \cdot E^{*}\right) \Delta_{\mu} \Delta_{\nu} \Delta_{\mu_{1}} \ldots \Delta_{\mu_{m}} P_{\mu_{m+1}} \ldots P_{\mu_{n}}\right] \\
& +B_{4, m}^{(n+2)}\left(\Delta^{2}\right) S\left[\left(\left(E^{\prime *} \cdot P\right) E_{\mu}+(E \cdot P) E_{\mu}^{\prime *}\right) P_{\nu} \Delta_{\mu_{1}} \ldots \Delta_{\mu_{m}} P_{\mu_{m+1}} \ldots P_{\mu_{n}}\right] \\
& +B_{5, m}^{(n+2)}\left(\Delta^{2}\right) S\left[\left(\left(E^{\prime *} \cdot P\right) E_{\mu}-(E \cdot P) E_{\mu}^{* *}\right) \Delta_{\nu} \Delta_{\mu_{1}} \ldots \Delta_{\mu_{m}} P_{\mu_{m+1}} \ldots P_{\mu_{n}}\right] \\
& +\frac{B_{6, m}^{(n+2)}\left(\Delta^{2}\right)}{M^{2}} S\left[(E \cdot P)\left(E^{\prime *} \cdot P\right) P_{\mu} P_{\nu} \Delta_{\mu_{1}} \ldots \Delta_{\mu_{m}} P_{\mu_{m+1}} \ldots P_{\mu_{n}}\right] \\
& \left.+\frac{B_{7, m}^{(n+2)}\left(\Delta^{2}\right)}{M^{2}} S\left[(E \cdot P)\left(E^{\prime *} \cdot P\right) \Delta_{\mu} \Delta_{\nu} \Delta_{\mu_{1}} \ldots \Delta_{\mu_{m}} P_{\mu_{m+1}} \ldots P_{\mu_{n}}\right]\right\} \\
& +\bmod (n, 2) B_{4, n}^{(n+2)}\left(\left(E^{\prime *} \cdot P\right) E_{\mu}+(E \cdot P) E_{\mu}^{\prime *}\right) \Delta_{\nu} \Delta_{\mu_{1}} \ldots \Delta_{\mu_{n}} .
\end{aligned}
$$

This differs from the original equation in the addition of the final term which appears only for odd $n$.

The unnumbered equation in Appendix B should be replaced by

$$
\begin{aligned}
& \left\langle p^{\prime} E^{\prime}\left|S\left[\tilde{G}_{\mu \alpha} i \stackrel{\leftrightarrow}{D}{ }_{\mu_{1}} \ldots i \stackrel{\leftrightarrow}{D}{ }_{\mu_{n}} G_{\mu_{n+1}}^{\alpha}\right]\right| p E\right\rangle=\sum_{\substack{m \text { even } \\
m=0}}^{n+1} \tilde{B}_{1, m}^{(n+2)}\left(\Delta^{2}\right) S\left[\epsilon_{\mu \alpha \beta \gamma} E^{\alpha} E^{\prime * \beta} P^{\gamma} \Delta_{\mu_{1}} \ldots \Delta_{\mu_{m}} P_{\mu_{m+1}} \ldots P_{\mu_{n+1}}\right] \\
& +\sum_{\substack{m \text { even } \\
m=0}}^{n} \tilde{B}_{2, m}^{(n+2)}\left(\Delta^{2}\right) S\left[\epsilon_{\mu \alpha \beta \gamma} E^{\alpha} E^{\prime * \beta} \Delta^{\gamma} \Delta_{\mu_{1}} \ldots \Delta_{\mu_{m+1}} P_{\mu_{m+2}} \ldots P_{\mu_{n+1}}\right] \\
& +\sum_{\substack{m \text { even } \\
m=0}}^{n} \tilde{B}_{3, m}^{(n+2)}\left(\Delta^{2}\right) S\left[\left(\epsilon_{\mu \alpha \beta \gamma} E^{\alpha} P^{\beta} \Delta^{\gamma} E_{\mu_{n+1}}^{*}+\epsilon_{\mu \alpha \beta \gamma} E^{\prime * \alpha} P^{\beta} \Delta^{\gamma} E_{\mu_{n+1}}\right) \Delta_{\mu_{1}} \ldots \Delta_{\mu_{m}} P_{\mu_{m+1}} \ldots P_{\mu_{n}}\right] \\
& +\sum_{\substack{m \text { even } \\
m=0}}^{n+1} \frac{\tilde{B}_{4, m}^{(n+2)}\left(\Delta^{2}\right)}{M^{2}} S\left[\epsilon_{\alpha \beta \gamma \delta} E^{\alpha} E^{\prime * \beta} P^{\gamma} \Delta^{\delta} \Delta_{\mu} \Delta_{\mu_{1}} \ldots \Delta_{\mu_{m}} P_{\mu_{m+1}} \ldots P_{\mu_{n+1}}\right] .
\end{aligned}
$$

Here, the T-parity has been corrected, which makes $\tilde{B}_{5, m}^{(n+2)}$ redundant, and the GFFs formerly labeled as $\tilde{B}_{\{6,7\}, m}^{(n+2)}$ have been eliminated since they are redundant under application of the Schouten identities [1] (note that this decomposition is consistent with Ref. [1] after application of those identities). 
Equation (8) should be replaced by

$$
\begin{aligned}
& \left\langle p^{\prime} E^{\prime}\left|S\left[G_{\mu \mu_{1}} \stackrel{\leftrightarrow}{D}_{\mu_{3}} \ldots i \stackrel{\leftrightarrow}{D}_{\mu_{n}} G_{\nu \mu_{2}}\right]\right| p E\right\rangle=\sum_{\substack{m \text { even } \\
m=0}}^{n+1}\left\{A_{1, m-2}^{(n)}\left(\Delta^{2}\right) S\left[\left(P_{\mu} E_{\mu_{1}}-E_{\mu} P_{\mu_{1}}\right)\left(P_{\nu} E_{\mu_{2}}^{*}-E_{\nu}^{\prime *} P_{\mu_{2}}\right) \Delta_{\mu_{3}} \ldots \Delta_{\mu_{m}} P_{\mu_{m+1}} \ldots P_{\mu_{n}}\right]\right. \\
& +A_{2, m-2}^{(n)}\left(\Delta^{2}\right) S\left[\left(\Delta_{\mu} E_{\mu_{1}}-E_{\mu} \Delta_{\mu_{1}}\right)\left(\Delta_{\nu} E_{\mu_{2}}^{*}-E_{\nu}^{*} \Delta_{\mu_{2}}\right) \Delta_{\mu_{3}} \ldots \Delta_{\mu_{m}} P_{\mu_{m+1}} \ldots P_{\mu_{n}}\right] \\
& +A_{3, m-2}^{(n)}\left(\Delta^{2}\right) S\left[\left(\left(\Delta_{\mu} E_{\mu_{1}}-E_{\mu} \Delta_{\mu_{1}}\right)\left(P_{\nu} E_{\mu_{2}}^{* *}-E_{\nu}^{* *} P_{\mu_{2}}\right)-\left(\Delta_{\mu} E_{\mu_{1}}^{\prime *}-E_{\mu}^{\prime *} \Delta_{\mu_{1}}\right)\left(P_{\nu} E_{\mu_{2}}-E_{\nu} P_{\mu_{2}}\right)\right)\right. \\
& \left.\times \Delta_{\mu_{3}} \ldots \Delta_{\mu_{m}} P_{\mu_{m+1}} \ldots P_{\mu_{n}}\right] \\
& +A_{4, m-2}^{(n)}\left(\Delta^{2}\right) S\left[\left(E_{\mu} E_{\mu_{1}}^{* *}-E_{\mu_{1}} E_{\mu}^{* *}\right)\left(P_{\nu} \Delta_{\mu_{2}}-P_{\mu_{2}} \Delta_{\nu}\right) \Delta_{\mu_{3}} \ldots \Delta_{\mu_{m}} P_{\mu_{m+1}} \ldots P_{\mu_{n}}\right] \\
& +\frac{A_{5, m-2}^{(n)}\left(\Delta^{2}\right)}{M^{2}} S\left[\left((E \cdot P)\left(P_{\mu} \Delta_{\mu_{1}}-\Delta_{\mu} P_{\mu_{1}}\right)\left(\Delta_{\nu} E_{\mu_{2}}^{* *}-E_{\nu}^{\prime *} \Delta_{\mu_{2}}\right)\right.\right. \\
& \left.\left.+\left(E^{*} \cdot P\right)\left(P_{\mu} \Delta_{\mu_{1}}-\Delta_{\mu} P_{\mu_{1}}\right)\left(\Delta_{\nu} E_{\mu_{2}}-E_{\nu} \Delta_{\mu_{2}}\right)\right) \Delta_{\mu_{3}} \ldots \Delta_{\mu_{m}} P_{\mu_{m+1}} \ldots P_{\mu_{n}}\right] \\
& +\frac{A_{6, m-2}^{(n)}\left(\Delta^{2}\right)}{M^{2}} S\left[\left((E \cdot P)\left(P_{\mu} \Delta_{\mu_{1}}-\Delta_{\mu} P_{\mu_{1}}\right)\left(P_{\nu} E_{\mu_{2}}^{*}-E_{\nu}^{*} P_{\mu_{2}}\right)\right.\right. \\
& \left.\left.-\left(E^{\prime *} \cdot P\right)\left(P_{\mu} \Delta_{\mu_{1}}-\Delta_{\mu} P_{\mu_{1}}\right)\left(P_{\nu} E_{\mu_{2}}-E_{\nu} P_{\mu_{2}}\right)\right) \Delta_{\mu_{3}} \ldots \Delta_{\mu_{m}} P_{\mu_{m+1}} \ldots P_{\mu_{n}}\right] \\
& +\frac{A_{7, m-2}^{(n)}\left(\Delta^{2}\right)}{M^{2}}\left(E^{\prime *} \cdot E\right) S\left[\left(P_{\mu} \Delta_{\mu_{1}}-\Delta_{\mu} P_{\mu_{1}}\right)\left(P_{\nu} \Delta_{\mu_{2}}-\Delta_{\nu} P_{\mu_{2}}\right) \Delta_{\mu_{3}} \ldots \Delta_{\mu_{m}} P_{\mu_{m+1}} \ldots P_{\mu_{n}}\right] \\
& \left.+\frac{A_{8, m-2}^{(n)}\left(\Delta^{2}\right)}{M^{4}}(E \cdot P)\left(E^{\prime *} \cdot P\right) S\left[\left(P_{\mu} \Delta_{\mu_{1}}-\Delta_{\mu} P_{\mu_{1}}\right)\left(P_{\nu} \Delta_{\mu_{2}}-\Delta_{\nu} P_{\mu_{2}}\right) \Delta_{\mu_{3}} \ldots \Delta_{\mu_{m}} P_{\mu_{m+1}} \ldots P_{\mu_{n}}\right]\right\} \\
& +\sum_{\substack{m \text { odd } \\
m=3}}^{n}\left\{A _ { 9 , m - 2 } ^ { ( n ) } ( \Delta ^ { 2 } ) S \left[\left(P_{\mu} \Delta_{\mu_{1}}-\Delta_{\mu} P_{\mu_{1}}\right)\left(E_{\nu}^{\prime *} P_{\mu_{2}} E_{\mu_{3}}+E_{\nu} P_{\mu_{2}} E_{\mu_{3}}^{\prime *}-E_{\mu_{2}}^{\prime *} P_{\nu} E_{\mu_{3}}-E_{\mu_{2}} P_{\nu} E_{\mu_{3}}^{* *}\right)\right.\right. \\
& \left.\times \Delta_{\mu_{4}} \ldots \Delta_{\mu_{m+1}} P_{\mu_{m+2}} \ldots P_{\mu_{n}}\right] \\
& +\frac{A_{10, m-2}^{(n)}\left(\Delta^{2}\right)}{M^{2}}\left[\left(P_{\mu} \Delta_{\mu_{1}}-\Delta_{\mu} P_{\mu_{1}}\right)\left(E_{\nu}^{\prime *} \Delta_{\mu_{2}} E \cdot P-E_{\nu} \Delta_{\mu_{2}} E^{\prime *} \cdot P-E_{\mu_{2}}^{*} \Delta_{\nu} E \cdot P+E_{\mu_{2}} \Delta_{\nu} E^{\prime *} \cdot P\right)\right. \\
& \left.\times \Delta_{\mu_{3}} \ldots \Delta_{\mu_{m}} P_{\mu_{m+1}} \ldots P_{\mu_{n}}\right] \\
& +\frac{A_{11, m-2}^{(n)}\left(\Delta^{2}\right)}{M^{2}}\left[\left(P_{\mu} \Delta_{\mu_{1}}-\Delta_{\mu} P_{\mu_{1}}\right)\left(E_{\nu}^{\prime *} P_{\mu_{2}} E \cdot P+E_{\nu} P_{\mu_{2}} E^{\prime *} \cdot P-E_{\mu_{2}}^{\prime *} P_{\nu} E \cdot P-E_{\mu_{2}} P_{\nu} E^{\prime *} \cdot P\right)\right. \\
& \left.\left.\times \Delta_{\mu_{3}} \ldots \Delta_{\mu_{m}} P_{\mu_{m+1}} \ldots P_{\mu_{n}}\right]\right\} .
\end{aligned}
$$

As pointed out in Ref. [1], $A_{9, m}^{(n)}\left(\Delta^{2}\right), A_{10, m}^{(n)}\left(\Delta^{2}\right)$, and $A_{11, m}^{(n)}\left(\Delta^{2}\right)$, which appear for $n \geq 3$, were omitted previously. Additionally, there was a typographic error in the coefficient of $A_{7, m-2}^{(n)}$, and $A_{3, m}^{(n)}\left(\Delta^{2}\right)$ can be eliminated.

Note that the counting of the (nonminimal) displayed set of GFFs that is referenced after Eq. (3) in the main manuscript should be adjusted accordingly, and that these decompositions are not minimal, i.e., that redundancies enter at some $n$.

[1] W. Cosyn, A. Freese, and B. Pire, arXiv:1812.01511. 